\title{
Micotoxinas en la Salud Pública
}

\author{
Sandra Duarte-Vogel ${ }^{1}$ y Luis C. Villamil-Jiménez ${ }^{2}$ \\ ${ }^{1}$ Médica Veterinaria. Facultad de Medicina Veterinaria y Zootecnia. Universidad Nacional de Colombia. \\ E-mail: smduartev@unal.edu.co \\ ${ }^{2}$ Médico Veterinario. M. Sc. Ph. D. Facultad de Medicina Veterinaria y Zootecnia. Universidad Nacional \\ de Colombia. E-mail: 1cvillamilj@unal.edu.co
}

Recibido 20 Junio 2005/Enviado para Modificación 31 Diciembre 2005/Aceptado 14 Febrero 2006

\section{RESUMEN}

Las micotoxinas constituyen un problema en el ámbito mundial por su alta incidencia y niveles de ocurrencia en los alimentos para humanos y animales. Las condiciones de colonización de los sustratos por hongos micotoxigénicos así como su posterior contaminación con micotoxinas juegan un papel fundamental en las estrategias de vigilancia y control. Entre los principales hongos micotoxigénicos se encuentran los géneros Aspergillus spp., Penicillium spp. y Fusarium spp. Dentro de las familias más importantes de micotoxinas se encuentran: las aflatoxinas, los tricoticenos, la ocratoxina A, las fumonisinas y la zearalenona. Los diferentes mecanismos de acción tóxica de estas micotoxinas constituyen un riesgo para la salud humana y animal constituyéndose en una problemática de salud pública. En Colombia la situación es compleja dada la deficiente investigación al respecto, los estudios realizados en el país han demostrado que la contaminación de alimentos por algunas micotoxinas es significativa y que se deben formular políticas sanitarias para afrontar este limitante. Se discute el riesgo potencial de las micotoxinas para la salud pública, las dificultades en el diagnóstico y la legislación así como las implicaciones en la seguridad e inocuidad alimentaria.

Palabras Clave: Micotoxinas, salud pública, abastecimiento de alimentos, medicina veterinaria (fuente: DeCS, BIREME).

\section{ABSTRACT Micotoxins in Public Health}

Mycotoxins have become a worldwide problem due to their high incidence and levels of occurrence in human food and animal feed. The conditions for colonising substrates by mycotoxigenic fungus and later contamination by mycotoxins play an important role in surveillance and control strategies. The main mycotoxigenic funguses are the Aspergillus spp., Penicillium spp. and 
Fusarium spp genera, the main mycotoxins of interest for human health being aflatoxins, trichothecenes, ochratoxin $A$, fumonisins and zearalenone. These mycotoxins' toxic action mechanisms constitute a risk for both human and animal health, causing diseases in both populations. The situation in Colombia is complex due to the lack of research having been carried out; the few studies made to date have demonstrated the high levels of contamination of food and feed in the country. This paper discusses mycotoxins' potential risk to public health, the difficulties involved in diagnosis and legislation and suggests policy implications for food safety.

Key Words: Mycotoxins, public health, food supply, veterinary medicine (source: $\mathrm{MeSH}, \mathrm{NLM}$ ).

$\mathrm{L}$

as micotoxinas son metabolitos secundarios producidos por diferentes géneros y especies de hongos, dentro de los cuales los principales son Aspergillus spp., Fusarium spp. y Penicillium spp, éstos colonizan y contaminan sustratos que son utilizados en la alimentación humana y animal, se estima que el $25 \%$ de la producción mundial de cereales se encuentra contaminada (1).

Desde el siglo VII A. C. se conocen los efectos de la colonización por hongos en los cultivos y se han relacionado brotes de enfermedades en humanos y animales con el consumo de los alimentos contaminados con micotoxinas (2); desde de 1960, con el advenimiento de la micotoxicología moderna se han descrito varias micotoxinas; las más importantes por sus implicaciones en salud humana y animal son las aflatoxinas, los tricoticenos, la ocratoxina A, las fumonisinas y la zearalenona $(1,3)$. Los efectos tóxicos de las micotoxinas sobre la salud humana y animal son de curso crónico, incluyen carcinogenicidad, inmunosupresión y disrupciones endocrinas $(4,5)$; la principal vía de exposición es la oral a través del consumo de alimentos contaminados, se presentan casos de micotoxicosis por inhalación (6-8).

\section{Aflatoxinas (AF)}

Producidas por hongos del género Aspergillus spp. $(1,9,10)$, dentro del grupo de aflatoxinas la de mayor importancia en salud pública es la aflatoxina $B_{1}$ $\left(\mathrm{AFB}_{1}\right)$, ésta se relaciona con el desarrollo de carcinoma hepatocelular en poblaciones que consumen alimentos contaminados (1). Su mecanismo de acción tóxica, involucra la formación de ligandos entre uno de sus metabolitos, producido en el hígado luego de la ingestión de la toxina, con el ADN de los hepatocitos, causando alteraciones en su replicación $(11,12)$ esta clasi- 
ficada dentro del grupo I según la IARC, por su efecto carcinogénico para humanos y animales $(13,14)$.

El carcinoma hepatocelular es una de las causas de muerte por cáncer en especial en regiones de Asia y África, originándose estudios epidemiológicos que buscan correlacionar la presentación del carcinoma con el consumo de aflatoxinas y la interacción de éstas con otras enfermedades hepáticas $(2,7,15)$.

La $\mathrm{AFM}_{1}$, se origina en el hígado de los mamíferos luego del consumo de alimentos contaminados con $\mathrm{AFB}_{1}$. Está clasificada según la IARC dentro del grupo $2 \mathrm{~B}$ como posible carcinógeno para humanos $(13,14)$ este metabolito se excreta en la leche y puede ser ingerido por los humanaos al consumir este alimento contaminado, siendo la población infantil la más expuesta $(3,16)$. Aunque es claro que el órgano blanco de las aflatoxinas es el hígado, se han encontrado evidencias de inmunosupresión por exposición a estas micotoxinas $(1-3,17)$.

Tricoticenos (TCT)

Son producidos por varias especies del género Fusarium spp.; son de distribución mundial y contaminan cereales como avena, trigo, cebada y maíz $(18,19)$.

Los TCT tienen múltiples órganos blanco, causando lesiones y alteraciones de características muy variadas (18) siendo la inmunosupresión, la neurotoxicidad y la disminución en la absorción de nutrientes las principales alteraciones $(1,3,19)$. El mecanismo de toxicidad de los tricotocenos, es la inhibición de la síntesis de proteínas, así como de la síntesis de ADN y ARN (18), por la unión de los TCT a la peptidil transferasa, parte integral de la subunidad 60S del ribosoma $(3,19,20)$.

\section{Ocratoxina A (OTA)}

La ocratoxina A (OTA), es un producida por hongos de los géneros Aspergillus spp. y Penicillium spp. $(2,21)$, la distribución de esta micotoxina es mundial, contaminando sustratos como cereales, café, vino, jugo de uva y cerveza $(1,21,22)$. La inhibición de la síntesis de proteína se ha definido como el mecanismo de toxicidad primario para OTA, se da por la inhibición competitiva de fenilalanina-tARNPhe sintetasa, que detiene la elongación del péptido (23). 
Según la IARC la OTA se encuentra dentro del grupo 2B por ser posible carcinógeno para humanos $(13,14)$. Se ha encontrado relación entre el consumo de alimentos contaminados con OTA y la nefropatía endémica de los Balcanes $(1,2,24)$ y con la nefropatía porcina (1), determinando que el órgano blanco es el riñón $(25,26)$. Los efectos sobre el sistema inmune han sido evaluados observándose inmunosupresión marcada después de la administración oral de OTA $(1,3,27)$.

Fumonisinas

Son producidas por Fusarium verticillioides (sin. moniliforme) (28), fueron descubiertas en 1988. Se ha relacionado la fumonisina $\mathrm{B}_{1}\left(\mathrm{FB}_{1}\right)$ con la presentación de cáncer esofágico en humanos, leucoencefalomalacia en equinos y edema pulmonar porcino entre otros $(1,2,29)$.

Su distribución es mundial, siendo el maíz el cultivo más afectado (3). El mecanismo de acción tóxica de las fumonisinas involucra la inhibición de la enzima ceramida sintetasa (30), generando un acúmulo de las bases esfingiodes y una disminución de los esfingolípidos complejos $(31,32)$. Se encuentra clasificada según la IARC dentro del grupo 2B $(13,14)$.

\section{Zearalenona (ZEA)}

La zearalenona (ZEA) es una lactona ácida resorcílica producida por diferentes especies del género Fusarium spp. Es una micotoxina de distribución mundial y los principales sustratos afectados son trigo, maíz, sorgo, cebada $\mathrm{y}$ piensos (2).

La capacidad de la ZEA para acoplarse a los receptores del 17-ß-estradiol ha determinado la acción tóxica de esta micotoxina (1), que compite con los estrógenos por los receptores citosólicos de las células de los órganos blanco (33) y se une a estos, comportándose como un disruptor endocrino (34). En humanos se ha relacionado el consumo de ZEA con la presentación de pubertad precoz en niñas y aumento del tamaño de los órganos reproductores en niños (35).

\section{MICOTOXINAS EN COLOMBIA}

La situación actual en la investigación sobre micotoxinas en Colombia se encuentra resumida en la Tabla 1 . Se destaca la falta de desarrollo en el área 
y la importancia de crear mecanismos de vigilancia y control de los alimentos destinados para humanos y animales en cuanto al los niveles máximos permitidos de micotoxinas.

Tabla 1. Resumen de los estudios desarrollados en Colombia sobre contaminación con micotoxinas en sustratos destinados para el consumo humano y animal

\begin{tabular}{|c|c|c|c|c|c|}
\hline Micotoxina & Sustrato & $\begin{array}{l}\text { Muestras positivas/ } \\
\text { Muestras totales }\end{array}$ & $\begin{array}{l}\text { Mediana } \\
(\mathrm{ppb})\end{array}$ & $\begin{array}{l}\text { Promedio } \\
\text { (ppb) }\end{array}$ & $\begin{array}{c}\text { Rango de } \\
\text { contaminación } \\
(p p b)\end{array}$ \\
\hline $\begin{array}{l}\text { Aflatoxinas } \\
\text { (36) }\end{array}$ & $\begin{array}{l}\text { Maíz y } \\
\text { subproductos, } \\
\text { cereales, arroz y } \\
\text { subproductos, } \\
\text { pasabocas, semillas } \\
\text { de leguminosas }\end{array}$ & $22 / 248$ & $5,9 \mathrm{ng} / \mathrm{g}$ & $12,6 \mathrm{ng} / \mathrm{g}$ & $1,0-103,3 \mathrm{ng} / \mathrm{g}$ \\
\hline $\begin{array}{l}\text { Zearalenona } \\
\quad(37)\end{array}$ & $\begin{array}{l}\text { Materias primas y } \\
\text { alimentos } \\
\text { concentrados para } \\
\text { aves y cerdos }\end{array}$ & $60 / 200$ & $236 \mu \mathrm{g} / \mathrm{kg}$ & $436 \mu \mathrm{g} / \mathrm{kg}$ & $29-3956 \mu \mathrm{g} / \mathrm{kg}$ \\
\hline $\begin{array}{l}\text { Fumonisina } \\
\mathrm{B}_{1}(38)\end{array}$ & $\begin{array}{l}\text { Maíz y } \\
\text { subproductos }\end{array}$ & $78 / 120$ & $493 \mu \mathrm{g} / \mathrm{kg}$ & $234 \mu \mathrm{g} / \mathrm{kg}$ & $24-2964 \mu \mathrm{g} / \mathrm{kg}$ \\
\hline
\end{tabular}

\section{REFERENCIAS}

1. Mycotoxins: Risk in plant, animal and human systems. Council for Agricultural Science and Technology (CAST) 2003. Ames, Iowa. USA.

2. Periaca M, Radiç B, Lucić A, Pavlović M. Efectos tóxicos de las micotoxinas en el ser humano. Bulletin of the World Health Organization.1999; 77 (9): 754-766.

3. JECFA, Joint FAO/WHO Experts Committee on Food Additives. 2001; Vol 47.

4. Wild CP, Hall AJ. Epidemiology of mycotoxin-related disease. Human and Animal Relationships. Howard/Miller Eds. Berlin Heidelberg. 1996. pp 213226.

5. Frink-Gremmels J. Mycotoxins: their implications for human and animal health. The Veterinary Quarterly.1999; 21 (4): 115-120.

6. Jakab GJ, Hmieleski RR, Zarba A, Hemenway DR, Groopman JD. Respiratory aflatoxicosis: Suppression of pulmonary and systemic host defenses in rats and mice. Toxicology and Applied Pharmacology. 1994; 125: 198-205.

7. Groopman JD, Wang JS, Scholl P.Molecular biomarkers for aflatoxins: from adducts to gene mutations to human liver cancer. Canadian Journal of Physiology and Pharmacology. 1996; 74: 203-209. 
8. Krysińska-Traczyk E, Kiecoma I, Percowsky J, Dutkiewicz J. Levels of fungi and mycotoxins in samples of grain and grain dust collected in farms in eastern Poland. Ann Agric Environ Med. 2001; 8: 268-274

9. Tomašević-Canović M, Daković A, Rottinghaus G, Matijasěvić S, Đuričić M. Surfactant modified zeolites-new efficient adsorbents for mycotoxins. Micropourus and Mesopourus materials. 2003; 61:173-180

10. Vaamode G, Patriarca A, Fernández V, Comercio R, Degrossi C. Variability of aflatoxin and cyclopiazonic acid production by Aspergillus section flavi from different substrates in Argentina. International Journal of Food Microbiology. 2003; 88:79-84.

11. Wild CP, Turner PC. The toxicology of aflatoxins as a basis for public health decision. Mutagenesis. 2002; 17(6): 471-481.

12. Mela ME, Hamm ML, Henderson PT, Harris CM, Harris TM, Essigmann JM. The aflatoxin B1 formamiopyrimidine adduct plays a major role in causing the types of mutations observed in human hepatocelular carcinoma. PNA (Proceeding of National Academy of Science). 2002; 99(10): 6655-6660.

13. International Agency for Research of Cancer (IARC), 2002. Vol 82: 171, 301.

14. International Agency for Research of Cancer (IARC), 1993. Vol 56: 245, 489, 397-445, 467.

15. Egner P, Wang J-B, Zhu Y-R, Zhang B-C, Wu Y, Zhang Q-N et al. 2001. Chlorophyllin intervention reduces aflatoxin-DNA adducts in individuals at high risk for liver cancer. PNAS. 98(25):14601-14606.

16. Galvano F, Galofaro V, Galvano G. Occurrence and stability of aflatoxin M1 in milk and milk products: a worldwide review. Journal of food protection. 1996; 59(10): 1079-1090.

17. Methenitou G, Maravelias C, Athanaelis S, Dona A, Koutselinis A. Inmunomodulative effects of aflatoxins and selenium on human natural killer cells. Veterinary and Human Toxicology. 2001; 43 (4): 232-235.

18. Eriksen GS, Petterson H. Toxicological evaluation of trichothecenes in animal feed. Animal Feed Science and Technology. 2004; 114: 205-239.

19. Eriksen GS, Petterson H, LundhT. Comparative cytotoxicity of deoxynivalenol, their acetylated derivatives and de-epoxy metabolites. Food and Chemical Toxicology. 2004; 42: 619-624.

20. Larsen JC, Hunt J, Perrin I, Ruckenbauer P. Workshop on trichothecenes with a focus on DON: summary report. Toxicology Letters. 2004; 153: 1-22.

21. Ratola N, Martins L, Alves A. Ocrhatoxin A in wines-assessing global uncertainty associated with results. Analytica Chimica Acta. 2004; 513: 319-324.

22. Serra R, Mendosa C, Abrunhosa L, Pietri A, Venanacio A. Determination of ochratoxin in wine grapes: comparison of extraction procedures and method validation. Analytica Chimica Acta. 2004; 513: 41-47.

23. Assaf H, Azouri H, Pallardy M. Ochratoxin A induces apoptosis in human lymphocytes through down regulation of Bcl- $\mathrm{x}_{\mathrm{L}}$. Toxicological Sciences. 2004; 79:335-344. 
24. Urabcheva T, Petkova-Bocharova T, Grosso F, Nikolov I, Nicolay Chernozemky I, Castegnaro M, Dragacci S. Analysis of ochratoxin A in foods consumed by inhabitant from on area with Balkan Endemic Nephropathy: A 1 month follow-up study. Journal of Agriculture and Foood Chemistry. 2004; 52: 2404-2410.

25. Zepnik H, Völkel W, Dekant W. Toxicokinetics of the mycotoxin ochratoxin A in F344 rats after oral administration. Toxicology and Applied Pharmacology. 2003; 192: 36-44.

26. Müller G, Burket B, Rosner H, Köhler H. Effect of the mycotoxin ochratoxin A and some of its metabolites on human kidney cell lines. Toxicology in vitro. 2003;17:441-448

27. Lioi MB, Santoro A, Barbieri R, Salzano S, Ursini MV. Ochratoxin A and Zearalenone a comparative study on genotoxic effects and cell death induced in bovine lymphocytes. Mutation Research. 2004; 557:19-27.

28. Williams LD, Meredith FI, Riley RT. Fumonisin-ortho-phthalaldehyde derivate is stabilized at low temperature. Journal of Chromatography B. 2004; 806: 311-314.

29. Soriano JM, Dragacci S. Intake, decontamination and legislation of fumonisins in foods. Food Research International. 2004; 37: 367-374.

30. Enongene EN, Sharma RP, Bhandari N, Miller JD, Meredith FI, Voss KA, Riley RT.. Persistence and reversibility of the elevation in free sphingoid bases induced by fumonisin inhibition of ceramide synthase. Toxicological Sciences. 2002; 67: 173-181.

31. Westhuizen L, Gelderblom WC, Shephard GS, Swannevelder S. Disruption of sphingolipid biosyntesis in hepatocyte nodules: selective proliferative stimulus induced by fumonisin $\mathrm{B}_{1}$. Toxicology. 2004; 200: 69-75.

32. He Q, Kim J, Sharma RP. Silymarin protects against liver damage in BALB/c mice exposure to fumonisin $\mathrm{B}_{1}$ despite increasing accumulation of free sphingoid bases. Toxicological Sciences. 2004; 80: 335-342.

33. Withanage GS, Murata H, Koyama T. Agonistic and antagonistic effects of zearalenone, an estrogenic mycotoxin, on SKN, HHUA, and HepG2 human cancer cell lines. Veterinary and Human Toxicology. 2001; 43 (1) : 6-9

34. Osweiler GD. Mycotoxins: Contemporary issues of food animal health and productivity. Veterinary Clinics of North America: Food Animal Practice. $2000 ; 16(3): 511-530$

35. Abid-Essefi S, Baudrimont I, Hassen W, Ovanes Z, Mobio TA, Anane R, Crppy $\mathrm{EE}$, Bacha H. DNA fragmentation apoptosis and cell cycle arrest induced by zearalenone in cultured DOK, Vero and Caco-2 cells; prevention by vitamin E. Toxicology. 2003; 192: 237-248.

36. Diaz GJ, Perilla NS, Rojas Y. Ocurrence of aflatoxins in selected Colombian foods. Mycotoxin Research. 2001; 17: 15-20.

37. Diaz GJ, Céspedes AE. Natural occurrence of zearalenone in feeds and feedstuffs used in poultry and pig nutrition in Colombia. Mycotoxin Research. 1997; 13: 81-87.

38. Perilla NS, Diaz GJ. Incidence and levels of fumonisins contamination in Colombian corn and corn products. Mycotoxin Research. 1998; 14: 74-82. 